\title{
TRANSPARENCIA Y RENDICIÓN DE CUENTAS DE LOS MUNICIPIOS DE CASTILLA-LA MANCHA
}

\section{TRANSPARENCY AND ACCOUNTABILITY IN LOCAL GOVERNMENTS \\ OF CASTILLA-LA MANCHA (SPAIN)}

Recibido/Received: 25/02/2016

Modificado/Modified: 14/06/2016

Aceptado/Accepted: 6/09/2016

\author{
Juan Luis Manfredi Sánchez \\ Ana María López Cepeda \\ Miguel Álvarez-Peralta \\ Vanesa Saiz Echezarreta \\ José Reig Cruañes \\ Juan.Manfredi@uclm.es \\ Ana.LopezCepeda@uclm.es \\ Miguel.Alvarez@uclm.es \\ Vanesa.saiz@uclm.es \\ Jose.Reig@uclm.es
} Universidad de Castilla-La Mancha. Cuenca. España/Spain

\section{RESUMEN}

El proyecto de investigación GlobalCOM (CSO2013-46997-R) tiene como finalidad atender a la evolución del grado de transparencia en la comunicación pública digital local. Para ello, se evalúan 41 indicadores que atienden al grado de desarrollo del sistema de rendición de cuentas en los sitios web de ayuntamientos de toda España. La hipótesis de partida postula que las webs de los ayuntamientos españoles manifiestan importantes deficiencias a la hora de dar cuenta de la gestión que realizan sus gobiernos. Ello afecta al papel que estas entidades juegan como fuentes informativas para los medios de comunicación y la ciudadanía, dificultando el debate público y generando desconfianza.

Esta comunicación presenta los resultados de la primera oleada de datos, procedentes de 33 municipios de más de 10.000 habitantes en Castilla-La Mancha, durante el primer cuatrimestre del año 2015. Los resultados confirman la hipótesis para la muestra analizada.

PALABRAS CLAVE

Infoparticip@, Transparencia, periodismo, innovación, comunicación local.

\section{SUMARIO}

1. Introducción. 2. Metodología. 3. Ranking de Transparencia municipal en CLM. 4. Puntuación media de las webs de los ayuntamientos de Castilla-La Mancha en relación a la provincia en que se ubican. 5. Cumplimiento de los indicadores analizados. 6. Conclusiones y recomendaciones. Bibliografía. 


\begin{abstract}
The research project GlobalCOM (CSO2013-46997-R) addresses the level of transparency in public local digital communication. To this effect, it evaluates 41 indicators related with the system of accountability on the websites of each council. The hypothesis claims that the websites of Spanish local governments show major deficiencies associated with the lack of transparency about the local administration processes. This have a negative impact on the role that these institutions play as information sources for the media and the citizens, hampering public debate and generating mistrust against local politics.

This paper presents the results of the first set of results obtained from 33 municipalities with more than 10,000 inhabitants within Castilla-La Mancha, during the first quarter of 2015. The results confirm the hypothesis for the selected sample.
\end{abstract}

\title{
KEYWORDS
}

Infoparticip@, transparency, journalism, innovation, local administration.

\section{CONTENTS}

1. Introduction. 2. Methodology. 3. City Councils Transparency ranking at CLM. 4. Average mark of the municipalities' web sites. 5. Grade of fulfillment. 6. Conclusions and recommendations. References.

\section{INTRODUCCIÓN}

El mapa Infoparticip@ analiza y evalúa cómo se utilizan las tecnologías digitales de las webs municipales a través de 41 indicadores propios. El objetivo final es el fomento de las buenas prácticas en materia de comunicación e información en las corporaciones municipales, así como la mejora entre los representantes políticos, los técnicos municipales y los periodistas para, finalmente, facilitar la participación ciudadana en la gestión política municipal.

El proyecto inicial incorporaba datos de seis comunidades autónomas introducidos por investigadores de nueve universidades. Liderado por la doctora Amparo Moreno Sardà, directora del Laboratorio de Periodismo y Comunicación para la Ciudadanía Plural (LPCCP) de la Universidad Autónoma de Barcelona (UAB), se trata de un proyecto que forma parte de una investigación financiada por el Ministerio de Economía y Competitividad para el período 2013-2015, titulado Comunicación y periodismo para la participación ciudadana en el seguimiento y evaluación de la gestión de los gobiernos locales. Al Mapa Infoparticip@ se le han añadido datos de los municipios de Castilla-La Mancha, Castilla-León, Murcia, Comunidad Valenciana, Asturias, Cantabria, Extremadura, Islas Baleares, Navarra y La Rioja, como parte del trabajo del grupo investigador Comunicación pública, transparencia, rendición de cuentas y participación en los gobiernos locales, bajo la dirección del profesor Juan Luis Manfredi Sánchez de la Facultad de Periodismo de la Universidad de Castilla-La Mancha, campus de Cuenca, se trata de un proyecto que forma parte de una investigación financiada por el Ministerio de Economía y Competitividad para el período 2014/2016.

El resultado del proyecto Mapa Inforpaticip@ es un mapa interactivo de España vinculado a una base de datos y otros recursos gráficos secundarios. Las evaluaciones son públicas, se someten a revisiones periódicas y persiguen la mejora de la información pública disponible.

El análisis de la información de las web se basa en 41 indicadores derivados de cuatro preguntas básicas para las cuales los ciudadanos tienen derecho a tener respuesta: quiénes son los representantes políticos, cómo gestionan los recursos colectivos, cómo informan de la gestión, y qué herramientas ofrecen para la participación ciudadana en el control 
democrático. Todas ellas se inscriben en la creciente preocupación política, periodística y ciudadana por la transparencia de las instituciones, que en tiempos recientes se ha convertido en uno de los temas recurrentes en la investigación en el área de Estructura de Medios (Manfredi Sánchez y Cabezuelo Lorenzo, 2015:41).

Los resultados se publican en el Mapa Infoparticip@ y se representan de acuerdo con un infómetro que traduce la puntuación que ha obtenido cada web en un color: blanco, si no ha logrado el 25\% de indicadores positivos; amarillo, si ha conseguido entre el $25 \%$ y el $50 \%$; y verde, si supera este porcentaje. De este modo, cualquier persona puede contrastar los datos, hacer aportaciones al equipo de investigación y reclamar a los responsables políticos de su municipio las mejoras que considere oportunas. Este proyecto ha dado lugar ya en el pasado a diversos volúmenes recogiendo los primeros resultados y discutiendo diferentes valoraciones metodológicas (Molina Rodríguez-Navas, 2015).

De acuerdo con la Ley 19/2013 del 9 de diciembre, de transparencia, acceso a la información pública y buen gobierno, los municipios deben adecuar su estrategia de información pública a las obligaciones contenidas en dicha ley. La normativa continúa lo establecido en la Ley 7/1985, de 2 de abril, Reguladora de las Bases del Régimen Local. En su Artículo 69.1, establece lo siguiente:

1. Las Corporaciones locales facilitarán la más amplia información sobre su actividad y la participación de todos los ciudadanos en la vida local.

2. Las formas, medios y procedimientos de participación que las Corporaciones establezcan en ejercicio de su potestad de auto-organización no podrán en ningún caso menoscabar las facultades de decisión que corresponden a los órganos representativos regulados por la Ley.

La investigación encuentra acomodo en la literatura académica en base a la creciente acreditación de la expansión de las tecnologías de la información como oportunidad para mejorar la gestión pública, recuperar la confianza de los ciudadanos y estimular la opinión pública crítica (Criado y Ramilo, 2003; Moon, Lee y Roh, 2014). Por eso es necesario que el ciudadano pueda conocer cómo actúan los poderes públicos, qué responsabilidades tienen en el ejercicio de dicho poder, y pueda preguntar libremente sobre aquello que le parezca oportuno para formarse su propia opinión, a partir de la consideración de la información pública de calidad como derecho ciudadano (De-Miguel-Molina, 2010).

Para Healey (1996) la difusión de la información refleja los valores sociales e identifica las preferencias de la ciudadanía. Para Cegarra-Navarro, "e-government is useful for the discovery of citizen needs in real time (live)" (2012:475). En un momento de reducción de ingresos y ajuste presupuestario, la ausencia de información reduce la capacidad de evaluación de la gestión (Zafra-Gómez et al. 2015). La decisión política de recortar los servicios, la subida de impuestos o establecer prioridades de gobierno son decisiones estratégicas que requieren información pública (Levine, 1978).

En el plano cívico, una sociedad desinformada se expone al descrédito generalizado de la política y del periodismo. La comunicación pública debe comprender que el tamaño de las poblaciones no importa, sino la capacidad de ofrecer información concreta de interés público en cuatro ejes:

1. Indicadores sobre quiénes son los representantes políticos

2. Indicadores sobre cómo gestionan los recursos colectivos (Gandía y Archidona, 2008);

3. Indicadores sobre cómo informan de la gestión de los recursos colectivos (CegarraNavarro et al., 2012:470);

4. Indicadores sobre qué herramientas ofrecen para la participación ciudadana en el control democrático (Gaventa, 2004). 


\section{METODOLOGÍA}

\subsection{Hipótesis}

Las dos hipótesis de partida básicas son las siguientes. En primer lugar, las webs de los ayuntamientos españoles ponen de manifiesto deficiencias a la hora de dar cuenta de la gestión que realizan los gobiernos, que afectan al papel que estas entidades juegan como fuentes de información de los medios de comunicación, enturbiando y en ocasiones contaminando el debate público, y generando desconfianza en la ciudadanía.

La hipótesis 1 sería la siguiente: "La cantidad de la información pública disponible en las webs municipales es escasa."

En segundo término, las tecnologías digitales pueden colaborar a reducir estas deficiencias y, así, a despertar un renovado interés entre la ciudadanía en la valoración de la actividad política y el control democrático. Sin embargo, para ello, los periodistas y otros responsables de la comunicación y la información de las administraciones públicas han de cambiar sus rutinas actuales, y han de ser capaces de utilizar las tecnologías digitales para elaborar la información de manera que resulte fácilmente inteligible a la ciudadanía plural.

La hipótesis 2 se redacta así: "La tecnología permite una mejora sustancial de la información pública disponible. La transparencia informativa y la rendición de cuentas son procesos de mejora constante"

De las hipótesis nace el diseño de la investigación. El objetivo general del proyecto es la investigación de los recursos, los rasgos y las prácticas de la comunicación pública que realizan los ayuntamientos a través de la web. Este análisis nos permitirá conocer cómo se facilita (o debilita) la participación ciudadana en el seguimiento y la evaluación de la gestión de los gobiernos locales, se explorarán las posibilidades de las tecnologías digitales. El segundo objetivo general es la publicación de los resultados de la investigación en tiempo real en el Mapa Infoparticip@. El análisis de los municipios de las diez comunidades autónomas antes señaladas.

\subsection{Objetivos}

Los objetivos concretos son los siguientes:

1. Identificar en las webs de los ayuntamientos de las Comunidades Autónomas anteriormente señaladas cuáles son los recursos y las prácticas de información, comunicación y periodismo, así como las deficiencias que se ponen de manifiesto, para dar cuenta de la gestión que realizan los gobiernos municipales.

2. Evaluar estos recursos y prácticas de acuerdo con un repertorio de indicadores que permiten valorar la comunicación local pública y el grado de transparencia con que se da cuenta de la gestión que realizan los gobiernos municipales.

3. Hacer un análisis cualitativo de la información que se ofrece en estas webs sobre la gestión del gobierno, para calibrar en qué casos se ha hecho, o no se ha hecho, un tratamiento periodístico de manera que informaciones complejas resulten fácilmente comprensibles a la ciudadanía.

\subsection{Procedimiento y muestra}

El proyecto de investigación se gesta con anterioridad a la ley de transparencia que rige en España y de la legislación posterior de las comunidades autónomas. Por eso, el diseño de la investigación es original y distinta a cualquier otro conjunto de indicadores sobre transparencia, buen gobierno o comunicación local. El criterio de creación y redacción de los indicadores es el siguiente: la información tiene que ser pública y accesible. Al mismo 
tiempo, la información tiene que estar publicada en una sección adecuada, no escondida, siguiendo la idea de "máximo tres clics". La nomenclatura contribuye o disminuye la transparencia informativa de la web.

Los indicadores básicos se han creado y organizado a partir de cuatro ejes informativos, que se definen de la siguiente manera: 1) el primero es el perfil de los representantes políticos, información sobre su trayectoria y actividad profesional, 2) el segundo grupo responde a la gestión de recursos colectivos a través de los órganos correspondientes, 3) el tercer punto evalúa la comunicación y la información pública y 4) el último eje se organiza en torno a la participación de la ciudadanía en la vida municipal.

La recogida de la información se hace de forma manual, esto es, cada investigador tiene un número limitado de ayuntamientos asignados y cuenta con unos criterios homogeneizados de validación. Para eso, hemos diseñado cursos de formación de evaluadores.

Se debe visitar la web, registrar la información y copiar los enlaces correspondientes. Este método solo permite la alternativa Sí/No. O bien se cumple el criterio completo o bien no se cumple y se marca negativamente. La disyuntiva evita otras valoraciones personales o los criterios particulares. El investigador completa la ficha y se envía a un segundo evaluador antes de publicar los resultados en el mapa.

Cada publicación en el mapa se convierte en un ítem geolocalizado, que hemos denominado "infómetro". El color del ítem señala el número de indicadores positivos. El color es blanco si no alcanza el 25\%; el color es amarillo si no alcanza el 50\%; el color es verde si no alcanza el $75 \%$; el color es verde con un punto cuando supera el $75 \%$.

El mapa es una representación gráfica de los resultados, que facilita la comprensión entre todo tipo de públicos. La identificación con un ítem permite al visitante obtener la ficha completa del municipio. La información incluye la información: número de habitantes, puntuación, indicadores superados y ficha de evaluación.

Figura 1. Ejemplo de interfaz del mapa. (Fuente: Mapa Infoparticip@)

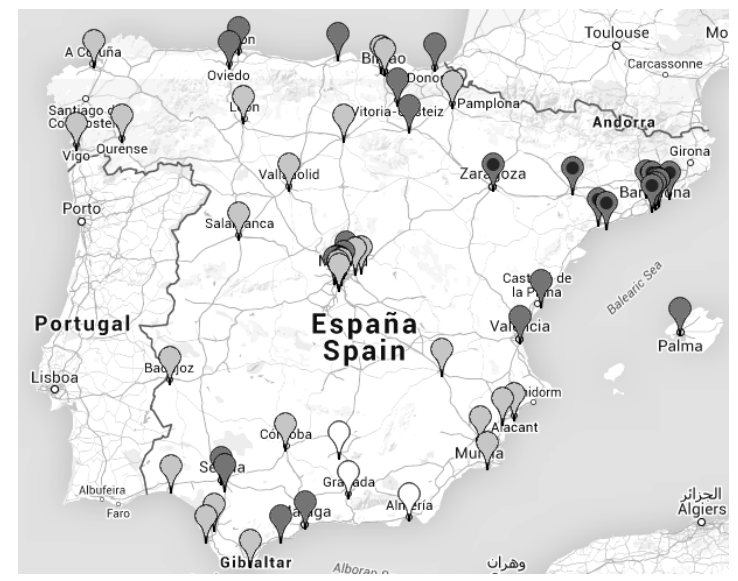

La naturaleza pública de la ficha permite al lector, al periodista o al responsable municipal identificar sus carencias, corregir algún error o establecer informes parciales. El mapa permite la búsqueda de datos según determinados criterios objetivos: comunidad autónoma, franja de número de habitantes, capitalidad, sexo del alcalde, partido político. 
El equipo responsable del Mapa Infoparticip@ está formado por profesores de la Facultad de Periodismo, que ha realizado la primera ola de evaluación con datos procedentes de 33 municipios castellano-manchegos de más de 10.000 habitantes, repartidos en las cinco provincias, durante el período comprendido entre enero y abril de 2015. Los resultados actuales están disponibles en la web www.mapainfoparticipa.com, que se irá actualizando ante cada nueva oleada de datos recogidos.

\section{RANKING DE TRANSPARENCIA MUNICIPAL EN CLM}

Si España está en el puesto $37^{\circ}$ del ranking mundial en transparencia de los poderes públicos es en parte porque la aprobación de la ley 19/2013, de 9 de diciembre, de transparencia, acceso a la información pública y buen gobierno ha hecho que mejore su puesto, ya que antes estaba en el número 64 (Matas Pastor y Aguilera Povedano, 2015:364). Pero más allá de este indicador global ¿Cuál es el nivel de transparencia real en las administraciones más cercanas, los municipios?

El análisis de esta primera oleada en Castilla-La Mancha deja algunas impresiones negativas: sólo ocho de los ayuntamientos superan el aprobado en transparencia, de un total de 33. La media del conjunto se queda en un escaso 39,76\% de indicadores positivos.

Lo primero que llama la atención en el ranking general por municipios es que los primeros puestos son ocupados por las capitales de provincia, con la excepción de Cuenca. Quizá lo que influye aquí no sea tanto la condición de capitalidad, sino el tamaño del municipio y, por ende, de los recursos que el Ayuntamiento puede destinar a aumentar la transparencia informativa. En tal caso, Cuenca vuelve a quedar desplazada desde la sexta posición que ostenta por número de habitantes hasta la novena que obtiene en el ranking de transparencia.

La media $(39,76)$ y la mediana $(36,59)$ de la escala de transparencia aparecen en posiciones contiguas, por lo que la distribución en el ranking resulta equilibrada, sin fuertes polarizaciones. Así se confirma en un primer examen: en los primeros puestos y bien destacadas del resto (puntuación mayor de 70), cuatro capitales de provincia muy pobladas. Las que les siguen en el tramo de transparencia de 50-60, Puerto Llano, Azuqueca, Valdepeñas y Talavera, son también poblaciones grandes (entre los 30.000 y los 55.000 habs.). Solo después aparece Cuenca, en el rango 40-50.

En el otro extremo (menos de 20 puntos de transparencia), los 3 municipios que ocupan la base de la escala (Caudete, Funsalida y Tarancón) pertenecen al rango inferior también por su número de habitantes, es decir, entre 10.000 y 20.000 habitantes censados.

Sin embargo, la relación entre tamaño y posición en el ranking no es del todo constante, ya que cinco de los municipios puntuados por encima de la media, se encuentran en el rango inferior por número de habitantes (Torrijos, El Casar, Manzanares, Campo de Criptana y Villacañas). Inversamente, algunos de los municipios más poblados aparecen por debajo de la media en el ranking de transparencia. Este es el caso, por ejemplo, de Hellín, Seseña, Illescas, Villarobledo, y Alcázar de San Juan.

Parece que la variable de tamaño de población no es muy decisiva, al menos, sin considerar otros factores como el de las mayorías políticas y el historial de alternancia en el poder. En cuanto a la variable de género, el porcentaje no es estadísticamente significativo para valorar si el sexo es un factor relevante en el cumplimiento de indicadores, habrá que esperar a completar la primera oleada en todas las comunidades para considerarlo en términos nacionales. Tan sólo seis municipios están gobernados por mujeres, entre ellos dos capitales de provincia Albacete y Ciudad Real. Las alcaldesas de Ciudad Real y Albacete 
ocupan el primer y cuatro puesto en el ranking de cumplimiento, mientras que las de Ocaña y Tarancón son el 25 y el 30 de la lista de 33 ayuntamientos estudiados, por lo que podríamos afirmar que sus puntuaciones responden a una distribución similar a la de sus compañeros varones.

En el cuadro adjunto aparecen los municipios ordenados por puntuación en indicadores de transparencia y a la derecha el número de habitantes y la posición ordinal en cuanto a población.

Cuadro 1. Ranking de Transparencia municipal en CLM

\begin{tabular}{|c|c|c|c|c|c|}
\hline & Ciudad & Provincia & $\begin{array}{l}\text { Índice } \\
\text { transpa- } \\
\text { rencia. }\end{array}$ & Habs. & Ranking habs. \\
\hline 1 & Ciudad Real & Ciudad Real & 75,61 & 74.960 & 5 \\
\hline 2 & Toledo & Toledo & 75,61 & 83.334 & 4 \\
\hline 3 & Guadalajara & Guadalajara & 73,17 & 83.720 & 3 \\
\hline 4 & Albacete & Albacete & 73,17 & 172.487 & 1 \\
\hline 5 & Puerto Llano & Ciudad Real & 56,1 & 50.608 & 7 \\
\hline 6 & Azuqueca & Guadalajara & 53,66 & 35.335 & 9 \\
\hline 7 & Valdepeñas & Ciudad Real & 53,66 & 30.705 & 12 \\
\hline 8 & Talavera de la Reina & Toledo & 53,66 & 86.779 & 2 \\
\hline 9 & Cuenca & Cuenca & 46,34 & 55.738 & 6 \\
\hline 10 & Tomelloso & Ciudad Real & 43,9 & 38.080 & 8 \\
\hline 11 & Torrijos & Toledo & 43,9 & 13.241 & 21 \\
\hline 12 & El casar & Guadalajara & 41,46 & 11.643 & 24 \\
\hline 13 & Manzanares & Ciudad Real & 41,46 & 18.924 & 17 \\
\hline 14 & Campo de Criptana & Ciudad Real & 41,46 & 14.387 & 20 \\
\hline 15 & Villacañas & Toledo & 41,46 & 10.089 & 33 \\
\hline \multicolumn{6}{|c|}{ Valor promedio: $\mathbf{3 9 , 7 6}$} \\
\hline 16 & Almansa & Albacete & 39,02 & 25.024 & 15 \\
\hline \multicolumn{6}{|c|}{ Valor mediana: 36,59} \\
\hline 17 & Consuegra & Toledo & 36,59 & 10.516 & 30 \\
\hline 18 & Madridejos & Toledo & 36,59 & 10.970 & 28 \\
\hline 19 & Alovera & Guadalajara & 34,15 & 12.237 & 22 \\
\hline 20 & Mora & Toledo & 34,15 & 10.315 & 31 \\
\hline 21 & Alcazar de San Juán & Ciudad Real & 31,71 & 31.650 & 10 \\
\hline 22 & Illescas & Toledo & 29,27 & 25.721 & 14 \\
\hline 23 & Villarobledo & Albacete & 26,83 & 26.419 & 13 \\
\hline 24 & Seseña & Toledo & 26,83 & 21.121 & 16 \\
\hline 25 & Ocaña & Toledo & 26,83 & 11.093 & 27 \\
\hline 26 & La Roda & Albacete & 24,39 & 15.979 & 18 \\
\hline 27 & Hellín & Albacete & 24,39 & 30.862 & 11 \\
\hline 28 & Yuncos & Toledo & 24,39 & 10.566 & 29 \\
\hline 29 & Sonseca & Toledo & 21,95 & 11.437 & 25 \\
\hline 30 & Quintanar de la Orden & Toledo & 21,95 & 11.987 & 23 \\
\hline 31 & Tarancón & Cuenca & 19,51 & 15.604 & 19 \\
\hline 32 & Caudete & Albacete & 19,51 & 10.294 & 32 \\
\hline 33 & Fuensalida & Toledo & 19,51 & 11.132 & 26 \\
\hline
\end{tabular}

Fuente. Elaboración propia a través de los datos publicados en Mapa Infoparticip@. 


\section{PUNTUACIÓN MEDIA DE LAS WEBS DE LOS AYUNTAMIENTOS DE CASTILLA-LA MANCHA EN RELACIÓN A LA PROVINCIA EN QUE SE UBICAN}

Si se realiza una comparativa entre las diferentes provincias se observa que, a diferencia de otras regiones, no existe una correlación directa entre provincias más pobladas y una mejor puntuación media de las webs provinciales o un mayor porcentaje de cumplimiento. Esta observación se diferencia de lo que ocurre con respecto a las capitales de provincia más pobladas tal y como se ha indicado en el epígrafe anterior.

Cuadro 2. Ranking de Transparencia por provincias en CLM

\begin{tabular}{|c|c|c|c|c|}
\hline & $\begin{array}{c}\text { Población } \\
\text { (INE 14) }\end{array}$ & Media provincial & $\begin{array}{c}\text { Mediana } \\
\text { provincial. }\end{array}$ & $\begin{array}{c}\text { Puntuación } \\
\text { capitales }\end{array}$ \\
\hline Toledo & 699.136 & 35,19 & 31,71 & 75,61 \\
\hline Ciudad Real & 519.613 & 49,3 & 43,9 & 75,61 \\
\hline Albacete & 396.987 & 34,55 & 25,61 & 73,17 \\
\hline Guadalajara & 255.426 & 50,61 & 47,56 & 73,17 \\
\hline Cuenca & 207.449 & 32,92 & 32,92 & 46,34 \\
\hline
\end{tabular}

Fuente. Elaboración propia a través de los datos publicados en Mapa Infoparticip@

La provincia más poblada (Toledo) ocupa el tercer lugar en el ranking de puntuación media, mientras que el ranking está liderado por la segunda menos poblada (Guadalajara). En cambio, sí podemos observar correlación entre la población de cada provincia y la puntuación obtenida por la web municipal de sus capitales.

En cuanto al grado de cumplimiento, todas las provincias tienen la mitad o más de las web analizadas (el 100\% en el caso de Guadalajara y Ciudad Real) por encima del 25\% de ítems cumplidos (lo que significaría al menos color amarillo en la señal del 'infómetro'), pero solo Guadalajara tiene la mitad de ellas por encima del 50\% de cumplimiento (señal verde en el 'infómetro'). Por tanto, tampoco se registra correlación entre población de cada provincia y cantidad de municipios que alcanzan categorías superiores de cumplimiento los ítems analizados.

Cuadro 3. Cumplimiento de Transparencia provincial en CLM

\begin{tabular}{|c|c|c|}
\hline & Municipios $>25 \%$ & Municipios $>50 \%$ \\
\hline Guadalajara & 100 & 50 \\
\hline Ciudad Real & 100 & 42,85 \\
\hline Albacete & 50 & 16,66 \\
\hline Toledo & 71,42 & 14,28 \\
\hline Cuenca & 50 & 0 \\
\hline
\end{tabular}

Fuente. Elaboración propia a través de los datos publicados en Mapa Infoparticip@

Las máximas puntuaciones coinciden siempre con las capitales de provincia, con la excepción de Cuenca, y en cuanto a las mínimas se encuentran muy repartidas, con tres poblaciones por debajo de los 20 ítems de cumplimiento distribuidas en diferentes provincias: Tarancón en Cuenca, Caudete en Albacete y Fuensalida en Toledo. 


\section{CUMPLIMIENTO DE LOS INDICADORES ANALIZADOS}

El análisis de los datos nos permite conocer el porcentaje de los ayuntamientos en Castilla-La Mancha que cumplen con cada uno de los indicadores que hemos dividido en cuatro grupos:

1. Indicadores sobre quiénes son los representantes políticos

2. Indicadores sobre cómo gestionan los recursos colectivos

3. Indicadores sobre cómo informan de la gestión de los recursos colectivos

4. Indicadores sobre qué herramientas ofrecen para la participación ciudadana en el control democrático

\subsection{Indicadores sobre quiénes son los representantes políticos}

Con respecto a esta primera categoría de indicadores, los ayuntamientos analizados en la primera oleada solo aprueban en lo que respecta a información básica sobre el alcalde o alcaldesa $(87,1 \%)$ y los miembros del gobierno $(60,7 \%)$, mientras que suspenden a la hora de informar sobre sus biografías o currículos, o sobre la composición de las fuerzas de oposición, donde no se alcanza el 50\%. Nuevamente destaca negativamente la provincia de Cuenca, si bien es necesario señalar que solo fueron analizados los dos únicos ayuntamientos que superaban los habitantes exigidos para esta primera oleada, lo que explica la tendencia a la polarización de sus puntuaciones.

Cuadro 4. Cumplimiento de los indicadores sobre quiénes son los representantes políticos

\begin{tabular}{|l|c|c|c|c|c|c|}
\hline $\begin{array}{l}\text { Indicadores sobre quiénes son los } \\
\text { representantes políticos }\end{array}$ & Albacete & $\begin{array}{c}\text { Ciudad } \\
\text { Real }\end{array}$ & Cuenca & Guadalajara & Toledo & Total \\
\hline $\begin{array}{l}\text { ¿Se da información básica sobre el } \\
\text { alcalde o la alcaldesa: nombre y } \\
\text { apellidos, foto y partido político? }\end{array}$ & $50 \%$ & $100 \%$ & $100 \%$ & $100 \%$ & $85,7 \%$ & $87,1 \%$ \\
\hline $\begin{array}{l}\text { iSe da información sobre el } \\
\text { alcalde o la alcaldesa: biografía } \\
\text { y/o currículo? }\end{array}$ & $16,7 \%$ & $57,1 \%$ & $0 \%$ & $50 \%$ & $28,6 \%$ & $30,5 \%$ \\
\hline $\begin{array}{l}\text { iSe da información básica sobre } \\
\text { los representantes que forman } \\
\text { parte del gobierno: nombres y } \\
\text { apellidos, fotos y partido político? }\end{array}$ & $50 \%$ & $57,1 \%$ & $50 \%$ & $75 \%$ & $71,4 \%$ & $60,7 \%$ \\
\hline $\begin{array}{l}\text { iSe da información sobre los } \\
\text { representantes que forman parte } \\
\text { del gobierno: biografía y/o } \\
\text { currículo? }\end{array}$ & $16,7 \%$ & $14,3 \%$ & $0 \%$ & $25 \%$ & $21,4 \%$ & $15,5 \%$ \\
\hline $\begin{array}{l}\text { iSe da información básica sobre } \\
\text { los representantes que no forman } \\
\text { parte del gobierno: nombres y } \\
\text { apellidos, fotos y partido político? }\end{array}$ & $33,3 \%$ & $57,1 \%$ & $0 \%$ & $50 \%$ & $35,7 \%$ & $35,2 \%$ \\
\hline $\begin{array}{l}\text { ¿Se da información sobre los } \\
\text { representantes que no forman parte } \\
\text { del gobierno: biografía y/o } \\
\text { currículo? }\end{array}$ & $16,7 \%$ & $14,3 \%$ & $0 \%$ & $25 \%$ & $14,3 \%$ & $14,1 \%$ \\
\hline
\end{tabular}

Fuente. Elaboración propia a través de los datos publicados en Mapa Infoparticip@ 


\subsection{Indicadores sobre cómo gestionan los recursos colectivos}

Al analizar los indicadores sobre cómo se gestionan los recursos colectivos, los porcentajes no son mucho mejores que en el cuadro anterior ya que los ayuntamientos de Castilla-La Mancha solo aprueban en cinco indicadores de un total de quince: información sobre la composición de los órganos de gobierno (56,4\%); publicación de las actas del Pleno Municipal (71,4\%); publicación de los acuerdos del gobierno o de la Junta de Gobierno Local (56,2\%); información sobre el Plan de Ordenación Urbanística (POUM) u otras normas de planificación urbanística (76,2\%); y publicación de las Ordenanzas Municipales $(97,1 \%)$. Salvo en este último caso, con unos resultados notables, sería recomendable que se mejorase en la transparencia del resto de los indicadores, en especial, de nuevo, en la provincia de Cuenca y, en menor medida, en Guadalajara.

Cuadro 5. Cumplimiento de los indicadores sobre gestión de recursos colectivos

\begin{tabular}{|c|c|c|c|c|c|c|}
\hline $\begin{array}{l}\text { Indicadores sobre cómo gestionan los } \\
\text { recursos colectivos }\end{array}$ & Albacete & $\begin{array}{c}\text { Ciudad } \\
\text { Real }\end{array}$ & Cuenca & Guadalajara & Toledo & Total \\
\hline $\begin{array}{l}\text { ¿Se da información sobre las competencias } \\
\text { de los órganos de gobierno: pleno, junta de } \\
\text { gobierno, comisiones informativas? }\end{array}$ & $33,3 \%$ & $42,6 \%$ & $0 \%$ & $75 \%$ & $35,7 \%$ & $37,3 \%$ \\
\hline $\begin{array}{l}\text { ¿Se da información sobre la composición } \\
\text { de estos órganos de gobierno? }\end{array}$ & $50 \%$ & $85,7 \%$ & $0 \%$ & $75 \%$ & $71,4 \%$ & $56,4 \%$ \\
\hline $\begin{array}{l}\text { ¿Se da información sobre el calendario de } \\
\text { trabajo de estos órganos de gobierno? }\end{array}$ & $16,7 \%$ & $28,6 \%$ & $0 \%$ & $0 \%$ & $0 \%$ & $9,1 \%$ \\
\hline $\begin{array}{l}\text { ¿Se publican las convocatorias con las } \\
\text { órdenes del día previas a la celebración de } \\
\text { los Plenos Municipales? }\end{array}$ & $16,7 \%$ & $42,6 \%$ & $0 \%$ & $75 \%$ & $28,6 \%$ & $32,6 \%$ \\
\hline $\begin{array}{l}\text { ¿Se publican las actas del Pleno } \\
\text { Municipal? }\end{array}$ & $50 \%$ & $85,7 \%$ & $50 \%$ & $100 \%$ & $71,4 \%$ & $71,4 \%$ \\
\hline $\begin{array}{l}\text { ¿Se publican los acuerdos del gobierno o } \\
\text { de la Junta de Gobierno Local? }\end{array}$ & $16,7 \%$ & $85,7 \%$ & $50 \%$ & $100 \%$ & $28,6 \%$ & $56,2 \%$ \\
\hline $\begin{array}{l}\text { ¿Se da información sobre el Plan de } \\
\text { Gobierno (PG), el Plan de Actuación } \\
\text { Municipal (PAM) y/o el Plan estratégico? }\end{array}$ & $0 \%$ & $28,6 \%$ & $0 \%$ & $0 \%$ & $7,1 \%$ & $7,1 \%$ \\
\hline $\begin{array}{l}\text { ¿Se da información sobre el Plan de } \\
\text { Ordenación Urbanística (POUM) u otras } \\
\text { normas de planificación urbanística? }\end{array}$ & $66,7 \%$ & $100 \%$ & $50 \%$ & $100 \%$ & $64,3 \%$ & $76,2 \%$ \\
\hline $\begin{array}{l}\text { ¿Se da información sobre otros planes } \\
\text { municipales: Agenda21, Juventud, } \\
\text { Participación ciudadana, etc.? }\end{array}$ & $33,3 \%$ & $71,4 \%$ & $50 \%$ & $50 \%$ & $7,1 \%$ & $42,4 \%$ \\
\hline $\begin{array}{l}\text { ¿Se publica la relación de puestos de } \\
\text { trabajo de la Corporación y las } \\
\text { retribuciones según las categorías? }\end{array}$ & $0 \%$ & $14,3 \%$ & $0 \%$ & $0 \%$ & $7,1 \%$ & $4,3 \%$ \\
\hline $\begin{array}{l}\text { ¿Se publican las retribuciones de los } \\
\text { cargos electos? }\end{array}$ & $16,7 \%$ & $14,3 \%$ & $0 \%$ & $25 \%$ & $21,4 \%$ & $15,5 \%$ \\
\hline ¿Se publican las Ordenanzas municipales? & $100 \%$ & $100 \%$ & $100 \%$ & $100 \%$ & $85,7 \%$ & $97,1 \%$ \\
\hline $\begin{array}{l}\text { ¿Se publica el Presupuesto del } \\
\text { Ayuntamiento? }\end{array}$ & $50 \%$ & $85,7 \%$ & $50 \%$ & $50 \%$ & $14,3 \%$ & $50 \%$ \\
\hline $\begin{array}{l}\text { ¿Se publica información sobre la ejecución } \\
\text { del Presupuesto? }\end{array}$ & $16,7 \%$ & $28,6 \%$ & $0 \%$ & $0 \%$ & $7,1 \%$ & $10,5 \%$ \\
\hline $\begin{array}{l}\text { ¿Se publican los informes anuales de la } \\
\text { Cuenta General y la Memoria de la gestión } \\
\text { económica de la Comisión Especial de } \\
\text { Cuentas? }\end{array}$ & $16,7 \%$ & $28,6 \%$ & $50 \%$ & $50 \%$ & $14,3 \%$ & $31,9 \%$ \\
\hline
\end{tabular}

Fuente. Elaboración propia a través de los datos publicados en Mapa Infoparticip@ 


\subsection{Indicadores sobre cómo informan de la gestión de los recursos colectivos}

El cumplimiento de los indicadores sobre cómo los municipios de Castilla-La Mancha informan de la gestión de los recursos colectivos muestra resultados muy desiguales. El $100 \%$ de los ayuntamientos analizados informa en sus páginas web del perfil del contratante y de las contrataciones y concesiones firmadas por la corporación con otras entidades, empresas o particulares, y prácticamente todos publican noticias locales en la web.

Muy distintos son los resultados obtenidos cuando se profundiza en el contenido de esas noticias ya que ninguno informa sobre las actuaciones de los miembros de la oposición relacionadas con el control de la gestión del gobierno, y solo el 15,7\% publica noticias en las que se contrastan las informaciones de miembros del gobierno con las de la oposición o los técnicos, en su caso. Ninguno de los municipios analizados informa sobre acuerdo del Pleno Municipal para apoyar el Decálogo de Buenas Prácticas de la Comunicación Local Pública, ni pública en la web dicho Decálogo lo que refleja un cierto desinterés por divulgar la importancia de la comunicación pública de proximidad.

Cuadro 6. Cumplimiento de los indicadores sobre cómo informan de la gestión de los recursos colectivos

\begin{tabular}{|l|c|c|c|c|c|c|}
\hline $\begin{array}{l}\text { Indicadores sobre cómo informan de } \\
\text { la gestión de los recursos colectivos }\end{array}$ & Albacete & $\begin{array}{c}\text { Ciudad } \\
\text { Real }\end{array}$ & Cuenca & Guadalajara & Toledo & Total \\
\hline ¿Se publican noticias en la web? & $83,3 \%$ & $100 \%$ & $100 \%$ & $100 \%$ & $100 \%$ & $96,7 \%$ \\
\hline $\begin{array}{l}\text { ¿Se publican noticias sobre las } \\
\text { actuaciones de los miembros del } \\
\text { gobierno relacionadas con el } \\
\text { rendimiento de cuentas? }\end{array}$ & $16,7 \%$ & $42,8 \%$ & $50 \%$ & $75 \%$ & $50 \%$ & $46,9 \%$ \\
\hline $\begin{array}{l}\text { ¿Se publican noticias sobre las } \\
\text { actuaciones de los miembros de la } \\
\text { oposición relacionadas con el control } \\
\text { de la gestión del gobierno? }\end{array}$ & $0 \%$ & $0 \%$ & $0 \%$ & $0 \%$ & $0 \%$ & $0 \%$ \\
\hline $\begin{array}{l}\text { ¿Se publican noticias en las que se } \\
\text { contrastan las informaciones de } \\
\text { miembros del gobierno, de la } \\
\text { oposición, y de técnicos en su caso? }\end{array}$ & $0 \%$ & $28,6 \%$ & $50 \%$ & $0 \%$ & $0 \%$ & $15,7 \%$ \\
\hline $\begin{array}{l}\text { ¿Se informa del perfil del contratante } \\
\text { y de las contrataciones y las } \\
\text { concesiones firmadas por la } \\
\text { Corporación con otras entidades, } \\
\text { empresas o particulares? }\end{array}$ & $100 \%$ & $100 \%$ & $100 \%$ & $100 \%$ & $100 \%$ & $100 \%$ \\
\hline $\begin{array}{l}\text { ¿Se informa sobre el acuerdo del } \\
\text { Pleno Municipal de apoyar el } \\
\text { Decálogo de Buenas Prácticas de la } \\
\text { Comunicación Local Pública? }\end{array}$ & $0 \%$ & $0 \%$ & $0 \%$ & $0 \%$ & $0 \%$ & $0 \%$ \\
\hline $\begin{array}{l}\text { ¿Se pública en la web el Decálogo } \\
\text { de Buenas Prácticas de la } \\
\text { Comunicación Local Pública? }\end{array}$ & $0 \%$ & $0 \%$ & $0 \%$ \\
\hline
\end{tabular}

Fuente. Elaboración propia a través de los datos publicados en Mapa Infoparticip@

\subsection{Indicadores sobre qué herramientas para la participación ciudadana en el control democrático se ofrecen a través del portal municipal}

Finalmente, en cuanto a los indicadores sobre qué herramientas ofrecen para la participación ciudadana en el control democrático, solo se supera el $50 \%$ en cinco de los trece indicadores analizados: 1) información básica sobre la situación del municipio y sus 
datos y actividades principales; 2) información histórica sobre el municipio; 3) redes sociales de la Corporación; 4) agenda de actividades municipales y ciudadanas; y 5) herramientas para comunicar incidencias de la vía pública, quejas o sugerencias.

Se observan deficiencias muy notables por parte de los municipios analizados a la hora de ofrecer herramientas de participación, de forma generalizada en todas las provincias analizadas.

Cuadro 7. Cumplimiento de los indicadores sobre qué herramientas ofrecen para la participación ciudadana en el control democrático

\begin{tabular}{|c|c|c|c|c|c|c|}
\hline $\begin{array}{l}\text { Indicadores sobre qué herramientas } \\
\text { ofrecen para la participación ciudadana en } \\
\text { el control democrático }\end{array}$ & Albacete & $\begin{array}{c}\text { Ciudad } \\
\text { Real }\end{array}$ & Cuenca & Guadalajara & Toledo & Total \\
\hline $\begin{array}{l}\text { ¿Se da información sobre la situación del } \\
\text { municipio: datos sobre el término } \\
\text { municipal, la población empadronada y la } \\
\text { diversidad social, actividades económicas, } \\
\text { culturales? }\end{array}$ & $66,7 \%$ & $71,4 \%$ & $50 \%$ & $75 \%$ & $14,3 \%$ & $55,5 \%$ \\
\hline $\begin{array}{l}\text { ¿Se da información histórica sobre el } \\
\text { municipio? }\end{array}$ & $71,4 \%$ & $71,4 \%$ & $100 \%$ & $100 \%$ & $85,7 \%$ & $85,7 \%$ \\
\hline $\begin{array}{l}\text { ¿Se ofrecen en la web las direcciones de } \\
\text { e-mail de los miembros del gobierno? }\end{array}$ & $33,3 \%$ & $57,1 \%$ & $50 \%$ & $50 \%$ & $28,6 \%$ & $43,8 \%$ \\
\hline $\begin{array}{l}\text { ¿Se ofrecen en la web las direcciones de } \\
\text { e-mail de los miembros de la oposición? }\end{array}$ & $16,7 \%$ & $28,6 \%$ & $50 \%$ & $25 \%$ & $14,3 \%$ & $26,9 \%$ \\
\hline $\begin{array}{l}\text { ¿Se ofrece en la web acceso a redes } \\
\text { sociales de la Corporación? }\end{array}$ & $66,7 \%$ & $71,4 \%$ & $100 \%$ & $75 \%$ & $78,6 \%$ & $78,3 \%$ \\
\hline $\begin{array}{l}\text { ¿Se da información sobre el reglamento } \\
\text { de participación ciudadana? }\end{array}$ & $50 \%$ & $14,3 \%$ & $0 \%$ & $25 \%$ & $14,6 \%$ & $20,8 \%$ \\
\hline $\begin{array}{l}\text { ¿Se da información en la web sobre otros } \\
\text { mecanismos de participación: consejos } \\
\text { territoriales, consejos de ciudad, consejos } \\
\text { sectoriales, etc.? }\end{array}$ & $16,7 \%$ & $42,8 \%$ & $0 \%$ & $50 \%$ & $28,6 \%$ & $27,6 \%$ \\
\hline $\begin{array}{l}\text { ¿Se ofrece en la web una agenda de } \\
\text { actividades municipales y ciudadanas? }\end{array}$ & $100 \%$ & $85,7 \%$ & $25 \%$ & $100 \%$ & $71,4 \%$ & $76,4 \%$ \\
\hline $\begin{array}{l}\text { ¿Se ofrece en la web el directorio de } \\
\text { entidades del municipio? }\end{array}$ & $33,3 \%$ & $71,4 \%$ & $0 \%$ & $75 \%$ & $28,6 \%$ & $41,7 \%$ \\
\hline $\begin{array}{l}\text { ¿Se ofrecen en la web herramientas de } \\
\text { participación para la elaboración y / o el } \\
\text { seguimiento del Plan de Gobierno, el Plan } \\
\text { de Actuación Municipal y / o el Plan } \\
\text { estratégico? }\end{array}$ & $0 \%$ & $0 \%$ & $0 \%$ & $0 \%$ & $0 \%$ & $0 \%$ \\
\hline $\begin{array}{l}\text { ¿Se ofrecen en la web herramientas de } \\
\text { participación para la elaboración y / o el } \\
\text { seguimiento del Presupuesto o de otros } \\
\text { planes municipales? }\end{array}$ & $0 \%$ & $14,3 \%$ & $0 \%$ & $0 \%$ & $7,1 \%$ & $4,3 \%$ \\
\hline $\begin{array}{l}\text { ¿Ofrecen a las ciudadanas y ciudadanos } \\
\text { herramientas para comunicar incidencias } \\
\text { de la vía pública, quejas o sugerencias? }\end{array}$ & $83,3 \%$ & $71,4 \%$ & $50 \%$ & $75 \%$ & $78,6 \%$ & $71,7 \%$ \\
\hline $\begin{array}{l}\text { Se publica el contacto con la persona } \\
\text { responsable de Prensa, Información y/o } \\
\text { Comunicación de la Corporación? }\end{array}$ & $0 \%$ & $14,3 \%$ & $0 \%$ & $25 \%$ & $14,3 \%$ & $10,7 \%$ \\
\hline
\end{tabular}

Fuente. Elaboración propia a través de los datos publicados en Mapa Infoparticip@ 


\section{CONCLUSIONES Y RECOMENDACIONES}

A partir del análisis pormenorizado que hemos expuesto, el presente análisis de la primera oleada de datos permite extraer las siguientes conclusiones generales:

- Sólo ocho de los ayuntamientos aprueban en transparencia, de un total de 33. La media del conjunto se queda en un escaso $39,76 \%$ de indicadores positivos.

- Los primeros puestos son acaparados por las capitales de provincia, con excepción de Cuenca. Esto puede deberse a que son los municipios más grandes y por consiguiente supuestamente con mayores recursos económicos como para disponer de personal especializado para la elaboración de su portal web.

- En el ranking por provincias, sin embargo, no son las provincias con más habitantes las que ofrecen mejores resultados, lo que podría indicar que las diputaciones no están destinando recursos a la mejora de la comunicación municipal.

- Las deficiencias más notables se observan en lo referente a herramientas de participación ofrecidas, y a la información detallada sobre los partidos de la oposición o los perfiles de los gobernantes.

- Se recomienda aprovechar los análisis detallados realizados para cada una de las webs para estudiar directamente en contacto con sus responsables en cada uno de los municipios cómo mejorar su transparencia y participación en cada uno de los indicadores analizados. Solo así se conseguirá una mayor nitidez, gobernanza y rendición de cuentas.

\section{BIBLIOGRAFÍA}

CEGARRA-NAVARRO, J. G.; CÓRDOBA PACHÓN, J.R. y MORENO CEGARRA, J. L. (2012). "E-Government and Citizens' Engagement with Local Affairs through E-Websites: The Case of Spanish Municipalities", International Journal of Information Management 32:469-478. http://doi:10.1016/j.ijinfomgt.2012.02.008

CLAVER-CORTÉS, E.; JUANA-ESPINOSA, S. y TARÍ, J.J. (2008). "E-Government Maturity at Spanish Local Levels", International Journal of Value Chain Management, 2(1): 4-17.

CRIADO, J.I. y RAMILO, M. C. (2003). "E-Government in Practice", International Journal of Public Sector Management, 16(3): 191-218. http://dx.doi.org/10.1108/09513550310472320.

DE-MIGUEL-MOLINA, M. (2010). "E-Government in Spain: An Analysis of the Right to Quality EGovernment", International Journal of Public Administration, 33(1):1-10. DOI: 10.1080/01900690 903178454

GANDÍA, J. L. y ARCHIDONA, M.C. (2008). "Determinants of Web Site Information by Spanish City Councils", Online Information Review, 32(1): 35-57. http://dx.doi.org/10.1108/1468452081086 5976

GAVENTA, J. (2004). "Strengthening Participatory Approaches to Local Governance: Learning the Lessons from Abroad", National Civic Review, 93(4): 16-27. DOI: 10.1002/ncr.67

GONZÁLEZ-BAILÓN, S. (2013). "Social Science in the Era of Big Data", Policy \& Internet 5(2):147160. DOI: $10.1002 / 1944-2866 . P O I 328$

HEALEY, P. (1996). "The Communicative Turn in Planning Theory and its Implications for Spatial Strategy Formation: Environment and Planning", Planning and Design 23(2): 217-234. DOI:10.1068/b230217

KARPF, D. (2012). "Social Science Research Methods in Internet Time”, Information, Communication \& Society, 15(5): 639-661. DOI: 10.2139/ssrn.1929095

LEVINE, C. (1978). "Organizational Decline and Cutback Management", Public Administration Review, 38(4): 316-325. 
MANFREDI SÁNCHEZ, J. L. y CABEZUELO LORENZO, F. (2015), "Innovación periodística para la rendición de cuentas", Cuadernos Artesanos de Comunicación, 78: 35-47.

MATAS PASTOR, J. y AGUILERA POVEDANO, M. (2015). "La transparencia en los gobiernos locales de las Islas Baleares", en R. Cetina Presuel, L. Corredoira y Alfonso y F. Gutiérrez Atala (Eds.). Medios y periodistas en la era del Gobierno Abierto y la Transparencia. Madrid: Universidad Complutense de Madrid, pp. 364-380.

MOLINA RODRÍGUEZ-NAVAS, P. (2015). Transparencia de la comunicación pública local. El Mapa Infoparticipa. La Laguna (Tenerife): Latina.

MOON, M. J.; LEE, J. y ROH, C.Y. (2014). "The Evolution of Internal IT Applications and EGovernment Studies in Public Administration: Research Themes and Methods", Administration \& Society, 46(1): 3-36. DOI: 10.1177/0095399712459723.

MORENO SARDÀ, A.; MOLINA RODRÍGUEZ-NAVAS, P.; CORCOY, M.; AGUILAR PÉREZ, A. y BORRÀS FARRAN, M. (2013). "Infoparticip@: periodismo para la participación ciudadana en el control democrático. Criterios, metodologías y herramientas", Estudios sobre el Mensaje Periodístico 19(2): 783-803. http://dx.doi.org/10.5209/rev_ESMP.2013.v19.n2.43471

NIELSEN, R. K. (2015). Local Journalism: The Decline of Newspapers and the Rise of Digital Media. Londres: IB Tauris.

ZAFRA GÓMEZ, J. L.; PLATA-DÍAZ, A. M.; PÉREZ LÓPEZ, G. y LÓPEZ HERNÁNDEZ, A. M. (2015). "Privatization of Waste Collection Services in Response to Fiscal Stress in Times of Crisis", Urban Studies, 2015: 1-20. DOI:10.1177/0275074008320710.

ZAFRA-GÓMEZ, J. L.; LÓPEZ-HERNÁNDEZ, A. M. y HERNÁNDEZ-BASTIDA, A. (2009). "Developing a Model to Measure Financial Condition in Local Government: Evaluating Service Quality and Minimizing the Effects of the Socioeconomic Environment: An Application to Spanish Municipalities", The American Review of Public Administration 39(4): 425-449. DOI:10.1177/ 0042098015586697. 


\section{Breve currículo:}

\section{Juan Luis Manfredi Sánchez}

Es doctor en Comunicación por la Universidad de Sevilla, donde obtuvo sendas licenciaturas en Periodismo e Historia. Es International Executive MBA por IE Business School, así como Máster en Gestión de Empresas Audiovisuales y en Administraciones Públicas (Universidad de Sevilla). Actualmente, colabora con el diario Cinco Días.

\section{Ana María López Cepeda}

Licenciada en Periodismo y Doctora en Comunicación y Periodismo por la Universidad de Santiago de Compostela (USC) (Premio Extraordinario de Doctorado de la Facultad de Ciencias de la Comunicación de la USC), y Licenciada en Derecho por la Universidad Nacional a Distancia (UNED). Trabaja como Profesora Contratada Doctora en la Facultad de Periodismo de la UCLM y es miembro del grupo de investigación Comunicación Pública: Poder, Derecho y Mensaje.

\section{Miguel Álvarez-Peralta}

Profesor Ayudante de Comunicación Política y Estructura de Medios en la Facultad de Periodismo UCLM. Doctor en Comunicación e Ingeniero Técnico en Informática de Sistemas por la Universidad Complutense de Madrid. Máster en Periodismo Científico por la UNED, donde impartió la asignatura Gabinetes de Prensa, y fue coordinador de divulgación científica. Miembro de la Junta Directiva de ULEPICC-España.

\section{Vanesa Saiz Echezarreta}

Profesora Contratada Doctora y Vicedecana de la Facultad de Periodismo de Cuenca en la UCLM. Especialista en Semiótica y Análisis Sociocultural de la Comunicación ha realizado la tesis "La solidaridad espacio de mediación de los sentimientos morales. Análisis de la publicidad de las ONGD 1999/2009)", en la UCM. Miembro del grupo de investigación Semiótica, Comunicación y Cultura (UCM), participa en el proyecto "Construcción de los Asuntos Públicos en la esfera pública mediatizada", ocupándose del análisis de la controversia sobre prostitución y trabajo sexual.

\section{José Reig Cruañes}

Profesor Ayudante Doctor en la Universidad de Castilla La Mancha. Licenciado en Historia, postgrado en Documentación; Documentalista de medios de comunicación en los 80 y primeros 90 en el Consell Assessor de RTVE de la Comunitat Valenciana. Doctorado (1999) Comunicación política en la transición democrática. Profesor en facultades de periodismo durante más de veinte años en Valencia y en Cuenca. La vertiente investigadora, con libros y artículos científicos sobre la prensa en el franquismo y la transición, sobre el espacio público en la globalización, comunicación electoral y estructura de los medios, se desarrolló en paralelo con las colaboraciones periodísticas en diversos medios. 